\title{
Humanizing critical care
}

\author{
VICTORIA BION ${ }^{1}$, HUGH MONTGOMERY ${ }^{2}$ \\ 1 Whittington Hospital NHS Trust, UK \\ 2 University College London, Whittington Hospital, UK \\ Corresponding author: \\ Victoria Bion \\ Institution for Sport, Exercise and Healt \\ University College London \\ 1st floor, 170 Tottenham Court Road \\ London W1T $7 H A$ \\ United Kingdom \\ Phone: 004407500335756 \\ E-mail:h.montgomery@ucl.ac.uk
}

\section{ABSTRACT}

Sleep is important for human neurocognitive, emotional and physical health. Increasing evidence shows that the intensive care unit environment is disruptive to sleep patterns. Such disruption is unpleasant to patients, but mounting evidence suggests that it may also worsen outcome. However, improvements in the patient experience are readily obtained through simple measures such as the use of eyepads and earplugs. Early data suggest that such interventions are not only kind, but may impact on patient outcomes such as delirium rates.

Key words: sleep, light, noise, sound, eyepad, earplug

Humans require sleep to maintain physical and mental health. There is certainly marked inter-individual variation in the duration of sleep necessary for optimal health, with genetic factors contributing between $31 \%$ and $55 \%$ of the variance. (1) But in addition to sleep quantity, the continuity and quality of sleep are important, as is its timing.

The human sleep circadian 'day' is a little over 24 hours, and sleep entrained by a series of cues: a daily change in light intensity (sunrise to sunset- or, at least, "light to dark'); changes in social and physical activity; patterns of food ingestion; and changes in light colour (from 'more blue' in the morning, to 'more red' in the evening. $(2,3)$ It is also readily disrupted or shifted, especially by nighttime light exposure: the cycle can be shifted by 12 hours in only 3 days. (3) 'Sleeping well' also requires the lack of other disruptive arousal stimuli (such as pain or discomfort, noise or bright lights), and a lack of other detrimental fac- tors (such as anxiety, or use of drugs such as beta-agonists or steroids). Such stimuli are common: noise levels on the ICU regularly exceed $80 \mathrm{~dB}$ - far more than recommended exposures (35dB at night, $45 \mathrm{~dB}$ during the day). (4)

One might thus expect sleep to be adversely impacted by the intensive care unit (ICU) environment, for a number of reasons. Exposure to natural daylight is not the norm. The light from monitors is in the 'blue' spectrum, sending a constant 'morning' cue to patients. Sleep entrainment is further weakened by the lack of social congress, lack of cyclical physical activity, and (often) the use of continuous enteral feeding in place of regular meals). Lights will go on at night in the context of new patient admissions, or for emergency or routine care (of the index patient or of others). Pain, discomfort or anxiety are all not uncommon, as is the use of drugs such as beta-agonists (whether for treatment of airways disease or for circulatory support). Evidence indeed suggests that sleep is adversely affected by the ICU environment. (5) Sleep disturbance affects the majority of ICU patients (6) and, indeed, can be near universal. (7) Sleep duration is reduced, it is no longer consolidated at night, is disrupted by frequent arousal events, and its architecture fragmented. (8) Environmental noise is responsible for many arousals (11.5\%) and awakenings (17\%) from sleep. (7) In one study of 7 mechanically ventilated patients, sound elevations occurred on average 36.5 times during each sleep hour, and caused $20.9 \%$ of total arousals and awakenings. (9) Patient care activities (average, 7.8 per hour of sleep) caused 7.1\%. (9)

Patients, relatives and clinical staff report this impact to be one of their great- est emotional stressors and least pleasant experiences (10), perhaps second only to pain. (8) Addressing the issue of 'sleeping badly' is thus essential, even if only on the grounds of compassion. However, poor sleep also influences systemic biological and physiological processes in a way which might also worsen 'hard outcomes'.

Even amongst healthy young male volunteers, six nights of reduced sleep (four hours each night) halved the mean IgG antibody response to influenza vaccine. (11) Similar impairments in the response to hepatitis A (12) or hepatitis B (13) vaccination have been reported. Experimental sleep restriction is also associated with impaired T-cell proliferation and natural killer cell activity, and with increased proinflammatory cytokine expression in white cells. The cellular immune response is also impaired in insomnia sufferers and nightshift workers - reviewed by Irwin (14). Susceptibility to the common cold after experimental inoculation is increased by sleep deprivation. (15)

Experimental sleep deprivation is associated with a rise in both blood pressure and heart rate, and chronic deprivation is associated with raised 24-hour blood pressure. Both deprivation and sleep disruption increase blood pressure variability and detrimentally affect diurnal cardiac output cycles, and may be associated with impaired salt handling - reviewed in (16). Such features may represent an increase in stress response: when compared to the situation after 12 hours in bed for 6 nights, sleep restricted young men (4 hours/night for 6 nights) exhibit raised sympathetic nervous system activity and evening circulating cortisol concentrations. (11) They also demonstrate impaired glucose tolerance, and reduced circulating thyrotropin con- 
centrations. (11) Others have confirmed such impacts, and have extended them to demonstrate a role of sleep deprivation in altering appetite - reviewed in (17).

This objectively observed increase in physiological stress can be associated with subjective changes in mood; The effects of sleep deprivation on increasing levels of anxiety are very well established. (18) Likewise, broad aspects of cerebral/neurocognitive function are detrimentally impacted by lack of (or disrupted pattern of) sleep (19): such effects include poor concentration and increased 'mind wandering' (20), impaired memory formation and consolidation (21), and impairment of global cognition. $(22,23)$

Pain sensitivity is increased by sleep deprivation: even sleeping 1.8 hours/night more than is habitual is associated with an increase in pain threshold. (24)

Simple measures can greatly improve sleep on ICU. Scotto compared the use of earplugs to routine clinical care in predominantly cardiological or general medical ICU patients (25), whilst $\mathrm{Hu}$ studied the use of ear plugs and eye masks both preand post-operatively with relaxing music before bed in cardiac surgical patients. (26) Sleep metrics (time taken to fall asleep, sleep duration, and number of awakenings) improved with intervention in both studies. Similarly, subjective sleep quality was improved by the use of earplugs with eyepads in cardiothoracic (27) or general ICU patients. (28)

Trials in ICU are lacking, but the limited data available from other sources do suggest that outcome might be affected by in- terventions designed to improve circadian entrainment and/or to improve sleep. In 89 post-spinal surgical 89 patients, those sited on the "bright/sunny" side (46\% higher light intensity) rather than "dim" side of the ward perceived less stress, and required $22 \%$ less analgesic medication per hour- effects independent of age. (29) Whether such effects were mediated through enhanced sleep was, however, not clear. However, in a study of 41 noncardiac surgical patients on the first night on the post-anaesthesia care unit, sleeping with eye pads and earplugs improved sleep quality and was associated with a reduced morphine requirement. (30)

In 628 patients admitted to a cardiac intensive care unit after a 1st myocardial infarction, length of stay was lower in women admitted to sunnier (south) rooms (2.3 vs 3.3 days), and mortality in both sexes consistently lower (21/293 [4.5\%]sunny vs 39/335 dull [11.6\%]). (31) Again, whether any effect was mediated through improved sleep was not clear.

Loss of natural circadian light rhythms may contribute to delirium (32), as may sleep disruption (above). When compared to 67 adult intensive care patients sleeping without earplugs, 69 randomised to earplug use demonstrated a lower incidence of confusion on the validated NEECHAM scale (Hazard Ratio 0.47) and reported better sleep. (33) In a general mixed ICU, earplug and eye mask use was associated with a reduced risk of delirium. (34) Metaanalysis suggests that the use of earplugs (alone or in combination with eyepads) significantly reduces the risk of delirium
(RR 0.77 ), and possibly even that of hospital mortality (RR 0.77). (35)

More recent studies have deployed multiple approaches to improve sleep. Li and colleagues studied the response of surgical ICU patients during a three-month intervention period that included dimming of lights between 23:00 and 05:00, lowering alarm volumes, and avoiding overnight investigations. (36) Sleep was improved and daytime somnolence reduced.

Patel, meanwhile, studied the impact of an intervention bundle to reduce noise, light, and nocturnal disruptions in a general mixed ICU. (34) Earplug and eye mask use was associated with improved sleep duration and quality and with reduced daytime drowsiness.

\section{CONCLUSION}

Sleep disruption on ICU is common and unpleasant, and may worsen outcome. There are many factors in the ICU environment which can be readily modified to address this issue. Such simple interventions are advocated.

\section{ACKNOWLEDGEMENTS}

Core funding for the author's place of work comes from the University College London Hospitals NHS Trust / University College London (UCLH/UCL) NIHR Comprehensive Biomedical Research Centre.

\section{REFERENCES}

1. Watson NF, Badr MS, Belenky G, et al. Recommended Amount of Sleep for a Healthy Adult: A Joint Consensus Statement of the American Academy of Sleep Medicine and Sleep Research Society. Sleep 2015; 38(6): 843-4.

2. Vandewalle G, Archer SN, Wuillaume C, et al. Effects of light on cognitive brain responses depend on circadian phase and sleep homeostasis. J Biol Rhythms 2011; 26(3): 249-59.

3. Duffy JF, Czeisler CA. Effect of Light on Human Circadian Physiology. Sleep Med Clin 2009; 4(2): 165-77.

4. Kamdar BB, Needham DM, Collop NA. Sleep deprivation in critical illness: its role in physical and psychological recovery. J Intensive Care Med 2012; 27(2): 97-111.

5. Weinhouse GL, Schwab RJ. Sleep in the critically ill patient. Sleep 2006; 29(5): 707-16.

6. Franck L, Tourtier JP, Libert N, Grasser L, Auroy Y. How did you sleep in the ICU? Crit Care 2011; 15(2): 408.

7. Freedman NS, Gazendam J, Levan L, Pack AI, Schwab RJ. Abnormal sleep/wake cycles and the effect of environmental noise on sleep disruption in the intensive care unit. Am J Respir Crit Care Med 2001; 163(2): 451-7.

8. Andersen JH, Boesen HC, Skovgaard Olsen K. Sleep in the Intensive Care Unit measured by polysomnography. Minerva Anestesiol 2013; 79(7): 804-15.

9. Gabor JY, Cooper AB, Crombach SA, et al. Contribution of the intensive care unit environment to sleep disruption in mechanically ventilated patients and healthy subjects. Am J Respir Crit Care Med 2003; 167(5): 708-15.

10. Novaes MA, Knobel E, Bork AM, Pavao OF, Nogueira-Martins LA, Ferraz MB. Stressors in ICU: perception of the patient, relatives and health care team. Intensive Care Med 1999; 25(12): 1421-6.

11. Spiegel K, Sheridan JF, Van Cauter E. Effect of sleep deprivation on response to immunization. JAMA 2002; 288(12): 1471-2. 
12. Lange T, Perras B, Fehm HL, Born J. Sleep enhances the human antibody response to hepatitis A vaccination. Psychosom Med 2003; 65(5): 831-5.

13. Prather AA, Hall M, Fury JM, et al. Sleep and antibody response to hepatitis B vaccination. Sleep 2012; 35(8): 1063-9.

14. Irwin MR. Sleep and infectious disease risk. Sleep 2012; 35(8): 1025-6.

15. Cohen S, Doyle WJ, Alper CM, Janicki-Deverts D, Turner RB. Sleep habits and susceptibility to the common cold. Arch Intern Med 2009; 169(1): 62-7.

16. Gangwisch JE. A review of evidence for the link between sleep duration and hypertension. Am J Hypertens 2014; $27(10)$ : 1235-42.

17. Copinschi G. Metabolic and endocrine effects of sleep deprivation. Essent Psychopharmacol 2005; 6(6): 341-7.

18. Pires GN, Bezerra AG, Tufik S, Andersen ML. Effects of acute sleep deprivation on state anxiety levels: a systematic review and metaanalysis. Sleep Med 2016; 24: 109-18.

19. Killgore WD. Effects of sleep deprivation on cognition. Prog Brain Res 2010; 185: 105-29.

20. Poh JH, Chong PL, Chee MW. Sleepless night, restless mind: Effects of sleep deprivation on mind wandering. J Exp Psychol Gen 2016; 145(10): 1312-8.

21. Havekes R, Vecsey CG, Abel T. The impact of sleep deprivation on neuronal and glial signaling pathways important for memory and synaptic plasticity. Cell Signal 2012; 24(6): 1251-60.

22. Maggio M, Colizzi E, Fisichella A, et al. Stress hormones, sleep deprivation and cognition in older adults. Maturitas 2013; 76(1): 22-44.

23. Gildner TE, Liebert MA, Kowal P, Chatterji S, Snodgrass JJ. Associations between sleep duration, sleep quality, and cognitive test performance among older adults from six middle income countries: results from the Study on Global Ageing and Adult Health (SAGE). J Clin Sleep Med 2014; 10(6): 613-21.

24. Roehrs TA, Harris E, Randall S, Roth T. Pain sensitivity and recovery from mild chronic sleep loss. Sleep 2012; 35(12): 1667-72.

25. Scotto CJ, McClusky C, Spillan S, Kimmel J. Earplugs improve patients' subjective experience of sleep in critical care. Nurs Crit Care 2009; 14(4): 180-4.

26. Hu RF, Jiang XY, Hegadoren KM, Zhang YH. Effects of earplugs and eye masks combined with relaxing music on sleep, melatonin and cortisol levels in ICU patients: a randomized controlled trial. Crit Care 2015; 19: 115.

27. Richardson A, Allsop M, Coghill E, Turnock C. Earplugs and eye masks: do they improve critical care patients' sleep? Nurs Crit Care 2007; 12(6): 278-86.

28. Jones C, Dawson D. Eye masks and earplugs improve patient's perception of sleep. Nurs Crit Care 2012; 17(5): 247-54.

29. Walch JM, Rabin BS, Day R, Williams JN, Choi K, Kang JD. The effect of sunlight on postoperative analgesic medication use: a prospective study of patients undergoing spinal surgery. Psychosom Med 2005; 67(1): 156-63.

30. Le Guen M, Nicolas-Robin A, Lebard C, Arnulf I, Langeron O. Earplugs and eye masks vs routine care prevent sleep impairment in post-anaesthesia care unit: a randomized study. Br J Anaesth 2014; 112(1): 89-95.

31. Beauchemin KM, Hays P. Dying in the dark: sunshine, gender and outcomes in myocardial infarction. J R Soc Med 1998; 91(7): $352-4$.

32. Wilson LM. Intensive care delirium. The effect of outside deprivation in a windowless unit. Arch Intern Med 1972; 130(2): 225-6.

33. Van Rompaey B, Elseviers MM, Van Drom W, Fromont V, Jorens PG. The effect of earplugs during the night on the onset of delirium and sleep perception: a randomized controlled trial in intensive care patients. Crit Care 2012; 16(3): R73.

34. Patel J, Baldwin J, Bunting P, Laha S. The effect of a multicomponent multidisciplinary bundle of interventions on sleep and delirium in medical and surgical intensive care patients. Anaesthesia 2014; 69(6): 540-9.

35. Litton E, Carnegie V, Elliott R, Webb SA. The Efficacy of Earplugs as a Sleep Hygiene Strategy for Reducing Delirium in the ICU: A Systematic Review and Meta-Analysis. Crit Care Med 2016; 44(5): 992-9.

36. Li SY, Wang TJ, Vivienne Wu SF, Liang SY, Tung HH. Efficacy of controlling night-time noise and activities to improve patients' sleep quality in a surgical intensive care unit. J Clin Nurs 2011; 20(3-4): 396-407. 